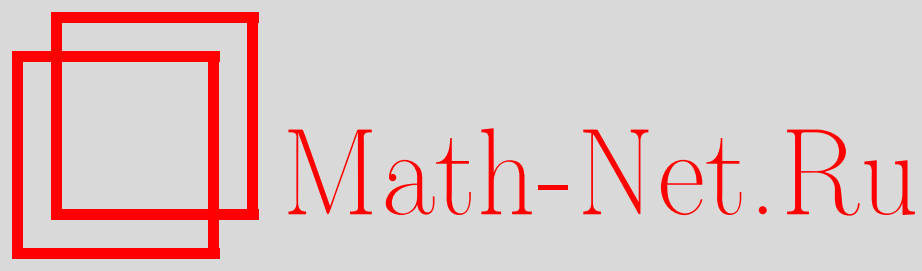

Ю. Г. Рудой, О. А. Котельникова, Функциональное уравнение для кроссовера в модели одномерных случайных блужданий Вейерштрасса, ТМФ, 2016, том 189, номер 3, 477-484

DOI: https://doi.org/10.4213/tmf9253

Использование Общероссийского математического портала Math-Net.Ru подразумевает, что вы прочитали и согласны с пользовательским соглашением http://www . mathnet.ru/rus/agreement

Параметры загрузки:

IP : 54.197 .217 .227

26 апреля 2023 г., 17:50:04

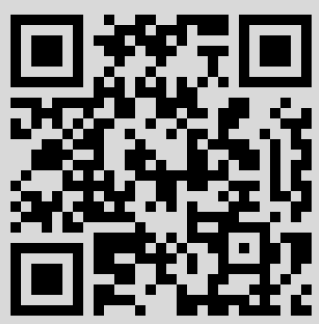




\title{
ФИЗИКА
}

Том 189, № 3

декабрь, 2016

(C) 2016 г. $\quad$ Ю. Г. Рудой* ${ }^{*}$ О.А. Котельникова ${ }^{\dagger}$

\section{ФУНКЦИОНАЛЬНОЕ УРАВНЕНИЕ ДЛЯ КРОССОВЕРА В МОДЕЛИ ОДНОМЕРНЫХ СЛУЧАЙНЫХ БЛУЖДАНИЙ ВЕЙЕРШТРАССА}

\begin{abstract}
Проблема одномерной симметричной диффузии рассмотрена в рамках подхода марковских случайных блужданий типа Вейерштрасса с использованием двухпараметрического скейлинга для переходной вероятности. Построено решение функционального уравнения для характеристической функции Ляпунова в виде суммы регулярного (однородного) и сингулярного (неоднородного) решений и найдены условия кроссовера от нормальной к аномальной диффузии.
\end{abstract}

Ключевые слова: нормальная и аномальная диффузия, марковский процесс, фрактальная размерность, функциональное уравнение, функция Вейерштрасса.

DOI: $10.4213 / \operatorname{tmf9253}$

\section{1. ВВЕДЕНИЕ}

Проблема диффузии - как нормальной, так и аномальной - продолжает оставаться одной из актуальных в современных физико-химических и иных приложениях (см., например, обзоры [1]--[3], где использован формализм дробных производных, недостаточно знакомый физикам). Кроме того, эта проблема представляет интерес и с математической точки зрения как один из разделов аналитической теории вероятностей (см., например, [4]-[7]). Связь между этими областями исследований основана на моделировании диффузионных процессов в рамках подхода одномерных (на непрерывной пространственно-координатной оси $-\infty<x<\infty$ ) и симметричных марковских случайных блужданий (СБ) с переходной вероятностью $P(x)$.

Особый интерес в этой связи представляют, например, работы [8], [9], где для симметричной функции $P(-x)=P(x)$ допускаются сколь угодно большие (в том числе

Работа выполнена при финансовой поддержке Министерства образования и науки РФ по Программе повышения конкурентоспособности РУДН среди ведущих мировых научно-образовательных центров на 2016-2020 гг.

* Российский университет дружбы народов, Москва, Россия. E-mail: rudikar@mail.ru

${ }^{\dagger}$ Московский государственный университет им. М.В. Ломоносова, Москва, Россия. E-mail: olga@magn.ru 
и бесконечные) значения эффективного радиуса $R_{\mathrm{eff}}=\sigma$. Здесь $\sigma^{2}=m_{2}$ - дисперсия, или второй центральный момент $P(x)$, причем для симметричного случая все четные центральные моменты совпадают с простыми моментами, а все нечетные моменты равны нулю.

Существенно, что в работах [8], [9] переходная функция $P(x)$ снабжается одним или двумя управляющими параметрами, что позволяет изменять $R_{\text {eff }}$ в широких пределах и тем самым переходить от нормальной диффузии (НД) с конечным значением $R_{\text {eff }}$ и экспоненциальным фронтом к аномальной диффузии (АД) с бесконечным $R_{\text {eff }}$ и степенным фронтом. Если начальное распределение $p_{0}(x)=\delta(x)$, то согласно [4]-[7] искомое распределение $p_{N}(x)$ после $N \geqslant 1$ шагов (скачков) представляется $N$-кратной сверткой функции $P(x)$, в связи с чем целесообразно перейти от функции $P(x)$ к ее фурье-образу $G(k)$ - характеристической функции Ляпунова, для которой свертка превращается в произведение. Кроме того, функция $G(k)$ сглаживает все особенности $P(x)$, а в силу тауберовых теорем интересующая нас асимптотика $p_{N}(x)$ как в дискретном времени (при $N \gg 1$ ), так и в пространстве (при больших значениях $x \gg 1$ ) определяется значениями $G(k)$ при малых значениях $k$ вблизи точки $k=0$.

Сложность этой задачи состоит в том, что для физически интересных функций $P(x)$ функции $G(k)$ часто являются весьма "экзотическими”, например обобщенная дзета-функция Римана для СБ Римана в работе [8] или функция Вейерштрасса ${ }^{1)}$ для СБ Вейерштрасса в работе [9]. Наибольший интерес представляет то обстоятельство, что в зависимости от значений управляющих параметров у названных функций имеются возможности как аналитического $\left(\sim k^{2}\right)$, так и неаналитического $\left(\sim k^{\alpha}, 0<\alpha<2\right)$ поведения $G(k)$ при $k \rightarrow 0$.

Существенно, что аналитическое поведение $G(k)$ универсально для всех функций $P(x)$ с конечным значением $R_{\text {eff }}=\sigma=\sqrt{m_{2}}$, причем согласно [4]-[7] $p_{N}(x)$ также имеет асимптотически универсальный вид, а именно нормальный (гауссовский) вид, и описывает НД Гаусса-Эйнштейна-Винера, где $p_{N}(x) \sim e^{-x^{2} / 2 \sigma_{N}^{2}}, \sigma_{N}^{2} \sim N$. По существу, это утверждение представляет собой один из вариантов формулировки центральной предельной теоремы [4].

При бесконечном $R_{\text {eff }}$, напротив, значение $\alpha$ специфично для каждой из функций $P(x)$ и различным образом зависит от входящих в $P(x)$ параметров; соответственно, фронт диффузии $p_{N}(x)$ имеет степенной вид $C_{\alpha} N x^{-(1+\alpha)}$, характерный для АД (иногда называемой также АД Леви-Хинчина). Ясно, что посредством управляющих параметров можно регулировать значение $R_{\text {eff }}=\sigma=\sqrt{m_{2}}$, тем самым реализуя кроссовер между двумя режимами - НД и АД - единого явления диффузии. Этот вопрос детально рассмотрен нами в работе [11] на основе аналогии диффузионного кроссовера с магнитным фазовым переходом.

В заключение этого раздела заметим, что даже явный вид функции $G(k)$, например заданной в виде бесконечного степенного или тригонометрического ряда, еще не дает возможности нахождения показателя $\alpha$. По-видимому, наиболее адекватным средством для этого является получение и решение функционального уравнения

1) Заметим, что в этом году исполняется ровно 100 лет со времени детального исследования этой функции, выполненного Харди в работе [10]. 
для $G(k)$ с использованием присущих этой функции свойств обобщенной однородности (самоподобия, масштабной инвариантности); этот подход широко используется, например, в методе ренормализационной группы (см., например, [12]).

Одним из преимуществ предлагаемого подхода является возможность избежать применения малознакомого физикам аппарата дробных производных [1]-[3]. В качестве содержательного примера рассмотрим далее модель СБ Вейерштрасса, сформулированную Монтроллом и др. в работе [9].

\section{2. МАРКОВСКИЕ СЛУЧАЙНЫЕ БЛУЖДАНИЯ ВЕЙЕРШТРАССА}

В работе [9] изучена простая, но достаточно содержательная модель для переходной вероятности $P(x)$ с двойным степенным масштабированием (иногда называемым скейлингом Бернулли):

$$
P(x, \lambda)=p \sum_{j=0}^{\infty} \lambda^{-j}\left[\delta\left(x-b^{j}\right)+\delta\left(x+b^{j}\right)\right] .
$$

В этой модели радиусъ одномерных шагов и соответствующие им вероятности образуют две неограниченные геометрические прогрессии: возрастающую (по длине шага) $\pm 1, \pm b, \ldots, \pm b^{j}, \ldots$, где $1<b<\infty$, и убывающую (по значению вероятности) $p, p / \lambda, \ldots, p / \lambda^{j}, \ldots$, где $1<\lambda<\infty, 0<p<1$. Число узлов $(j=0,1, \ldots)$ предполагается неограниченным, а значение $b>1$ для простоты в дальнейшем будем считать заданным $(b=\mathrm{const})$. Ясно, что с уменьшением $\lambda$ распределение $P(x, \lambda)$ становится все более широким (медленно убывающим), однако значения $\lambda \leqslant 1$ исключены ввиду нарушения условия нормировки, в силу которого $2\left[p+(p / \lambda)+\cdots+\left(p / \lambda^{j}\right)+\cdots\right]=1$, откуда легко найти $p=p(\lambda)=(1-1 / \lambda) / 2$.

Заметим, что в пределе $\lambda \rightarrow \infty$ в ряде (1) сохраняется лишь первое слагаемое с $j=0$, так что в этом пределе $P(x, \lambda) \rightarrow P(x, \infty)=P( \pm 1)=p(\infty)=1 / 2$, что совпадает со стандартным определением СБ Гаусса-Эйнштейна-Винера [4]-[7]; для этого случая распределение $P(x, \infty)$ является наиболее узким, причем $R_{\text {eff }}=\sigma=\sqrt{m_{2}}$ принимает минимальное значение, равное единице. Очевидно, что с уменьшением $\lambda$ распределение $P(x, \lambda)$ уширяется, так как величина $R_{\text {eff }}=\sigma=\sqrt{m_{2}}$ при этом возрастает - вплоть до ее расходимости, определяющей точку кроссовера между НД и АД. Для точной количественной оценки и нахождения указанной точки необходимо найти явное выражение для дисперсии $\sigma^{2}(b, \lambda)$, причем всегда $\partial \sigma^{2}(b, \lambda) / \partial \lambda<0$ при всех $\lambda$.

Удобным аппаратом для решения этой задачи является аппарат характеристической функции $G(k, \lambda)$, которая для $P(x, \lambda)$ из $(1)$ дается функцией Вейерштрасса

$$
G(k, \lambda)=2 p(\lambda) \sum_{j=0}^{\infty} \lambda^{-j} \cos \left(k b^{j}\right),
$$

причем $G(0, \lambda) \equiv 1$ в силу условия нормировки, $G^{\prime}(0, \lambda)=0$ в силу симметрии. Выражение (2) представляет собой тригонометрический ряд с пропусками (лакунами), позволяющий найти формальные выражения для всех четных моментов $m^{(2 s)}(\lambda)$, 
которые по определению равны производным $G^{(2 s)}(k, \lambda)$ по $k$ порядка $2 s$ при $k=0$ :

$$
\left|m_{2 s}(\lambda)\right|=2 p(\lambda) \sum_{j=0}^{\infty}\left(\frac{\lambda_{\mathrm{cr}}^{(s)}}{\lambda}\right)^{j}, \quad \lambda_{\mathrm{cr}}^{(s)}=\left(\lambda_{\mathrm{cr}}\right)^{s}, \quad \lambda_{\mathrm{cr}}=b^{2} .
$$

Если бы значения всех моментов (3) были конечны при любом значении $\lambda$, то можно было бы перейти от разложения Фурье (2) к разложению Тейлора для $G(k, \lambda)$, справедливому при всех значениях $1<\lambda<\infty$ (хотя бы вблизи точки $k=0$ ),

$$
G(k, \lambda)=1+\sum_{s=1}^{\infty} \frac{1}{(2 s) !}\left(i^{2 s}\right) m_{2 s}(\lambda) k^{2 s}
$$

Однако каждый момент (3) порядка $2 s$ имеет вид суммы бесконечной геометрической прогрессии со знаменателем $\lambda_{\mathrm{cr}}^{(s)} / \lambda$, где пороговые значения $\lambda_{\mathrm{cr}}^{(s)}$ образуют возрастающую (с ростом порядка $s$ от 1 до $\infty$ ) неограниченную геометрическую прогрессию со знаменателем $\lambda_{\mathrm{cr}}^{(s)}=\left(\lambda_{\mathrm{cr}}\right)^{s}, \lambda_{\mathrm{cr}}=b^{2}>1$.

Следовательно, каждый из рядов (3) для момента $m_{2 s}(\lambda)$ сходится только при $\lambda_{\text {cr }}^{(s)} / \lambda<1$, т. е. при достаточно больших значениях $\lambda$; только в этом случае моменты имеют конечные значения $m_{2 s}(\lambda)=2 p(\lambda)\left[1-\left(\lambda_{\text {cr }}^{(s)} / \lambda\right)\right]^{-1}$, где $p(\lambda)=(1-1 / \lambda) / 2$. Очевидно, что при любом конечном $\lambda$ в бесконечном ряде (4) всегда найдется бесконечное число расходящихся (сингулярных) слагаемых; именно это обстоятельство будет использовано ниже в разделе 3 при построении решения функционального уравнения для $G(k, \lambda)$ при любом $\lambda$.

Таким образом, ряд Тейлора (4) сходится только в рассмотренном выше пределе $\lambda \rightarrow \infty$, причем с учетом очевидного равенства $m_{2 s}(\infty)=2 p(\infty) \equiv 1$ для всех значений $s$ от 1 до $\infty$, сумма ряда (4) равна $\cos k$ при любых значениях $k$. Тот же результат, разумеется, следует и из разложения (2), в котором при $\lambda \rightarrow \infty$ остается лишь первое (при $j=0$ ) слагаемое $G(k, \infty)=\cos k$, так что режим НД вообще будет единственным для диффузии, а режим АД (и, следовательно, кроссовер АД/НД) будет отсутствовать в принципе.

В общем случае для произвольных значений $\lambda$ разложение Тейлора (4) теряет применимость и не может описывать все режимы диффузии. Иными словами, функция $G(k, \lambda)$ при конечных $\lambda$ не будет аналитической вблизи $k=0$, и ее разложение будет идти по нецелым степеням $k$, например слагаемое низшего порядка в этом разложении должно иметь вид $k^{\alpha}$, где $0<\alpha<2$, так что $m_{2}(\lambda) \equiv G^{(2)}(k=0, \lambda)$ будет тогда, как и ожидалось, расходиться при $\lambda<\lambda_{\mathrm{cr}}^{(1)}$.

Именно эти значения параметра $\lambda$ будут определять режим АД, но следует также учитывать, что при изменении $\lambda$ от бесконечно больших значений до минимального (равного единице) фактически будет происходить бесконечный каскад "кроссоверов", вполне аналогичных так называемой “чертовой лестнице" (“devil's staircase") фазовых переходов (см., например, [7]). Детальный анализ соответствующей фазовой диаграммы аналога магнитного фазового перехода с логарифмическим параметром порядка дан нами в работе [11], но здесь нас интересует взаимосвязь разложений (2) и (4). 


\section{3. ФУНКЦИОНАЛЬНОЕ УРАВНЕНИЕ ДЛЯ ФУНКЦИИ ВЕЙЕРШТРАССА}

Ввиду неприменимости разложения Тейлора (4) при любых конечных $\lambda$ необходимо предложить способ, позволяющий найти неаналитическую часть разложения исходной функции (2) при малых $k$. Монтролл и др. [9] использовали для этого представление зависящего от $k$ множителя $\cos \left(k b^{j}\right)$ в виде обратного преобразования Меллина с дальнейшим применением теоремы о вычетах. Как и следовало ожидать, в интервале значений $1<\lambda<\lambda_{\mathrm{cr}}^{(1)}=b^{2}$ имеет место разложение $G(k, \lambda) \approx 1-C_{\alpha} k^{\alpha}+\cdots$, где $\alpha=2 \ln \lambda / \ln \lambda_{\text {cr }}^{(1)}=\ln \lambda / \ln b$, так что $0<\alpha<2$ в этом интервале, причем $G^{\prime \prime}(0, \lambda)=m_{2}(\lambda)$, очевидно, расходится. Согласно [7], [9] показатель $\alpha$ определяет фрактальную размерность диффузионных кластеров.

Однако способ получения результата в работе [9] представляется физически не вполне прозрачным и требующим дополнительного математического обоснования, поэтому ниже предлагается альтернативный способ, основанный на существовании для $G(k, \lambda)$ функционального уравнения, вид которого следует непосредственно из определения (2):

$$
G(k, \lambda)=\frac{1}{\lambda} G(b k, \lambda)+2 p(\lambda) \cos k .
$$

Уравнение (5) является линейным неоднородным функциональным уравнением, справедливым во всем интервале значений $1<\lambda<\infty$, причем неоднородное слагаемое $2 p(\lambda) \cos k$ в правой части (5) регулярно по $k$ вблизи $k=0$ для всех допустимых значений $\lambda$. Отсюда ясно, что это слагаемое может давать вклад только в режим НД и, следовательно, режим АД (существующий только при $1<\lambda<b^{2}$ ) полностью обусловлен только однородным слагаемым $(1 / \lambda) G(b k, \lambda)$. С этим вполне согласуется тот факт, что слагаемое $(1 / \lambda) G(b k, \lambda)$ исчезает в предельном случае ${ }^{2)} \lambda \rightarrow \infty$, когда, как показано выше, существует только режим НД (при этом учтено, что значение $G(b k, \infty)=\cos (b k)$ всегда конечно).

На основе этих соображений нетрудно получить частное (сингулярное) решение $G_{\text {sing }}(k, \lambda)$ функционального уравнения $(5)$, описывающее АД при значениях $1<\lambda \leqslant$ $\lambda_{\text {cr }}=b^{2}$, полностью отбрасывая неоднородное регулярное слагаемое. Естественно искать $G_{\text {sing }}(k, \lambda)$ в виде ${ }^{3)}$

$$
G_{\text {sing }}(k, \lambda)=C(\lambda) k^{\alpha(\lambda)} Q(k), \quad Q(b k)=Q(k) .
$$

Подставляя (6) в усеченное (однородное) уравнение (5), легко получаем

$$
1=\left(\frac{1}{\lambda}\right) b^{\alpha(\lambda)}, \quad \alpha(\lambda)=\frac{\ln \lambda}{\ln b}, \quad 0<\alpha(\lambda) \leqslant 2, \quad 1<\lambda \leqslant \lambda_{\mathrm{cr}}=b^{2} .
$$

Таким образом, целесообразно искать полное решение уравнения (5) в виде суммы

$$
G(k, \lambda)=G_{\text {reg }}(k, \lambda)+G_{\text {sing }}(k, \lambda) .
$$

2)Это обстоятельство открывает возможность итерационного решения функционального уравнения (5) по степеням $1 / \lambda$.

3) Как будет показано ниже, множитель $Q(k)$ содержит только логарифмические по $k$ поправки. 
Возвращаясь к формальному разложению Тейлора (4), полностью регулярному только в пределе $\lambda \rightarrow \infty$, целесообразно при любом конечном $\lambda$ искать регулярную (аналитическую) часть решения $G(k, \lambda)$ в следующем виде (о выборе $S=S(\lambda)$ см. ниже; пока заметим лишь, что $S \rightarrow \infty$ при $\lambda \rightarrow \infty)$ :

$$
G_{\mathrm{reg}}(k, \lambda(S))=1+\sum_{s=1}^{S-1}(-1)^{s} \frac{1}{(2 s) !} m_{2 s}[\lambda(S)] k^{2 s} .
$$

Оставшаяся часть разложения (4) является сингулярной, или полностью неаналитической, благодаря рассмотренным выше расходимостям $m_{2 s}$ при $s \geqslant S$ :

$$
G_{\text {sing }}(k, \lambda(S))=1+\sum_{s=S}^{\infty}(i)^{2 s} \frac{1}{(2 s) !} m_{2 s}[\lambda(S)] k^{2 s} .
$$

Далее по аналогии с (6) бесконечную сумму (10) по целым (четным) степеням $k$ можно заменить всего одним слагаемым, но дробного порядка по $k$ :

$$
G_{\text {sing }}(|k|, \lambda(S))=C[\lambda(S)]|k|^{\alpha[\lambda(S)]} Q(|k|),
$$

где коэффициент $C[\lambda(S)]$ и аномальный показатель $\alpha[\lambda(S)]$ не зависят от $k$ (об амплитудной функции $Q(|k|)$ см. ниже). Заметим, что представление решения (8) в виде суммы (9) и (11) вполне аналогично часто используемой в приложениях формуле Тейлора в виде суммы многочлена Тейлора (9) и остаточного члена в форме (11), аналогичного форме Пеано ${ }^{4)}$, но с обратным следованием относительно порядкового символа $o$, так что $G_{\text {reg }}(k, \lambda(S))=o\left[G_{\text {sing }}(k, \lambda(S))\right]$. Иными словами, в определенном интервале значений $\lambda(S)$ (при заданном значении $S$ ) в интересующем нас пределе $k \rightarrow 0$ остаточный член превосходит основной, и потому именно он определяет асимптотическое поведение всей функции $G(k, \lambda)$, а тем самым и функции $P(x, \lambda)$ в пределе $x \rightarrow \infty$.

Фактическое построение разложений (9) и (11) основано на том, что для любого конечного значения $\lambda$ из открытого интервала $(1, \infty)$ всегда существует (также конечное) значение $S(\lambda)$ из полуоткрытого интервала $[1, \infty)$; соответственно, существует обратная функция $\lambda(S)$, для которой выполняются неравенства $\lambda_{\text {cr }}^{(S-1)}<$ $\lambda(S)<\lambda_{\mathrm{cr}}^{(S)}$, причем $\lambda_{\mathrm{cr}}^{(1)}=1, \lambda_{\mathrm{cr}}^{(2)}=b^{2}$ и т. д. Тогда моменты $m_{2 s}(\lambda)$ существуют и конечны только для $s=1,2, \ldots, S-1$ и расходятся для $s \geqslant S$, так что разложение (9) обрывается на слагаемом порядка $k^{2(S-1)}$.

Наибольший интерес представляет показатель $\alpha[\lambda(S)]$, удовлетворяющий строгому неравенству $2(S-1)<\alpha[\lambda(S)]<2 S$, так как только в этом случае все производные $G_{\operatorname{sing}}^{(2 s)}(k, \lambda)$ с $s \geqslant S$ становятся сингулярными при $k=0$. Действительно, пусть $2 S-\alpha=\varepsilon$ с $0<\varepsilon<2$, а $s=S+r, r=0,1, \ldots$. Тогда $G_{\text {sing }}^{(2 s)}(|k|, \lambda) \sim|k|^{-(\varepsilon+2 r)} \rightarrow \infty$ при $k=0$ и сколь угодно малом значении $\varepsilon>0$; что касается множителя $Q(|k|)$, то его не следует дифференцировать (см. сноску 3).

4) Заметим, что ни одна из других форм остаточного члена (Лагранжа, Коши, ШлёмильхаРоша) не подходит, поскольку подразумевает конечность производной более высокого порядка $G^{(2 S)}=m_{2 S}$, тогда как в нашем случае это условие нарушается вообще для всех значений $s \geqslant S$. 
Показатель $\alpha[\lambda(S)]$ можно вычислить точно - подобно тому, как это сделано в соотношении (8) с использованием только однородной части функционального уравнения (6) $G(|k|, \lambda)=(1 / \lambda) G(b|k|, \lambda)$, иногда называемом скейлинговым, или самоподобным; согласно этому уравнению $G(|k|, \lambda)$ является обобщением понятия однородной функции в смысле Эйлера. Предполагая дополнительно выполнение другого функционального уравнения $Q(b|k|)=Q(|k|)$ для амплитудной функции $Q(|k|)$, получаем из (9)

$$
1=\left[\frac{1}{\lambda(S)}\right] b^{\alpha[\lambda(S)]}, \quad \alpha[\lambda(S)]=\frac{\ln \lambda(S)}{\ln b} .
$$

Применим далее монотонную операцию логарифмирования к цепочке неравенств $\lambda_{\text {cr }}^{S-1}<\lambda(S)<\lambda_{\text {cr }}^{S}$ (все члены этой цепочки превышают единицу, так что их логарифмы положительны), а затем разделим почленно на $\ln \lambda_{\mathrm{cr}}>0$, откуда получим неравенство $S-1<\ln \lambda(S) / \ln \lambda_{\mathrm{cr}}<S$. Учитывая, что $\lambda_{\mathrm{cr}}=b^{2}$, окончательно приходим к неравенству $2(S-1)<\alpha[\lambda(S)]<2 S$, которое обеспечивает неаналитичность сингулярной части решения (11). Разделив найденное неравенство на $S$, заметим, что $2(1-1 / S)<\alpha[\lambda(S)] / S<2$, так что $\alpha / S \rightarrow 2$ при $S \rightarrow \infty, \lambda(S) \rightarrow \infty$, когда неаналитическая часть решения (11) (а вместе с ней АД) строго обращается в нуль (как показано в разделе 2).

Входящий в (11) коэффициент $C[\lambda(S)]$ можно определить с помощью “эвристических" соображений, причем результат полностью совпадает с результатом, полученным в работе [9] более строго посредством интегрального преобразования Меллина. Переход от первого расходящегося слагаемого порядка $2 S$, входящего в $G_{\mathrm{reg}}(k, \lambda)(9)$, к соответствующему слагаемому в $G_{\text {sing }}(|k|, \lambda)$ состоит в переходе между степенями $k$, а именно от $2 S$ к $\alpha<2 S$. Аналогичные замены проводятся и для коэффициента $1 /(2 S) !(i)^{2 S}$, переходящего в коэффициент $C(\alpha)$; именно, $1 /(2 S) ! \equiv 1 / \Gamma[1+(2 S) !]$ переходит в $1 / \Gamma[1+\alpha]=-(1 / \pi) \Gamma(-\alpha)$, тогда как множитель $(i)^{2 S} \equiv e^{[i(\pi / 2)]^{2 S}}$ переходит в $e^{[i(\pi / 2)]^{\alpha}}$, сохраняющий только четную часть $\cos [(\pi / 2) \alpha]$, которая обращается в нуль при любом четном значении $\alpha$.

Что касается амплитудной функции $Q(|k|)$, то для функционального уравнения $Q(b|k|)=Q(|k|)$ существует постоянное решение $Q=$ const (вообще не зависящее от $k$ ). В более общем случае указанное функциональное уравнение определяет лог-периодическую функцию, принадлежащую к классу медленно меняющихся функций (в смысле Карамата). Действительно, если мы проведем "логарифмическую" замену переменных $|k| \rightarrow \log (|k|), b \rightarrow \log b$ (с учетом условия $b>1$ ), то функциональное уравнение для $Q$ перейдет в более обычное функциональное уравнение для периодической функции $\mathbb{Q}(\log (|k|))=\mathbb{Q}(\log (|k|)+\log b)$.

Решение последнего уравнения имеет вид тригонометрического ряда Фурье по переменной $\log (|k|)$ с периодом $\log b$ :

$$
Q(|k|)=\frac{2}{\log b} \sum_{m=0}^{\infty} q_{m} \cos \left[2 \pi m \frac{\log (|k|)}{\log b}+\varphi_{m}\right],
$$

где амплитуды $q_{m}$ и начальные фазы $\varphi_{m}$ остаются неопределенными. Ряд (13) содержит только логарифмические по $|k|$ поправки $\sim[\log (|k|)]^{n}$, где $n$ - натуральные числа, что оправдывает утверждение, сделанное в сноске 4. 


\section{4. ВЫВОДЫ}

Проблема одномерной симметричной диффузии, в том числе кроссовер между режимами НД (Гаусса-Эйнштейна-Винера) и АД (Леви-Хинчина) рассмотрена на основе подхода марковских СБ. Показано, что в рамках модели Монтролла и др. [9] с двухпараметрическим скейлингом переходной вероятности для СБ Вейерштрасса можно найти сначала характеристическую функцию Ляпунова, а затем посредством тауберовых теорем и асимптотическое распределение вероятностей (или, иначе, фронт диффузии). Предложен конструктивный и физически прозрачный метод построения решения линейного неоднородного функционального уравнения, результаты которого полностью совпадают со строгим результатом, полученным ранее в работе [9] посредством интегрального преобразования Меллина.

\section{Список литературы}

[1] В. В. Учайкин, УФН, 173:8 (2003), 847-875.

[2] A. A. Dubkov, B. Spagnolo, V. V. Uchaikin, Intern. J. Bifur. Chaos Appl. Sci. Engrg., 18:9 (2008), 2649-2672.

[3] V. Zaburdaev, S. Denisov, J. Klafter, Rev. Modern Phys., 87:2 (2015), 483-530.

[4] В. Феллер, Введение в теорию вероятностей и ее приложения, 1, Мир, М., 1984.

[5] К. В. Гардинер, Стохастические методы в естественных науках, Мир, М., 1986.

[6] Н. Г. Ван Кампен, Стохастические процессы в физике и химии, Высшая школа, М., 1990.

[7] М. Шредер, Фракталь, хаос, степенные законы. Миниатюры из бесконечного рал, РХД, Ижевск, 2001.

[8] J.E. Gillis, G.H. Weiss, J. Math. Phys., 11:4 (1970), 1307-1312.

[9] B. D. Hughes, M. F. Shlesinger, E. W. Montroll, Proc. Natl. Acad. Sci. USA, 78:6 (1981), 3287-3291.

[10] G. H. Hardy, Trans. Amer. Math. Soc., 17:3 (1916), 301-325.

[11] Ю. Г. Рудой, Вестник Самарского госуд. ун-та. Сер. Матем., 1:66 (2008), 210-222.

[12] Д. В. Ширков, ТМФ, 60:2 (1984), 218-223.

Поступила в редакцию 18.07.2016, после доработки 1.08.2016 